\title{
LA DERNIÈRE RETRAITE DE JEAN-JACQUES
}

Au cours de son séjour chez le Marquis de Girardin, du 20 mai au 2 juillet 1778, Rousseau n'a pas écrit une ligne de son œuvre, correspondance comprise. Les quelques semaines qu'il passe à Ermenonville, avant d'y mourir, n'ont laissé aucune lettre. Nulle trace de cette ultime retraite. Les nombreux écrits et documents disponibles, tant des contemporains que des commentateurs, nous permettent cependant de reconstruire le contexte dans lequel il accepta l'hospitalité de René de Girardin, les motifs de cette dernière retraite, sa philosophie de l'asile et sa représentation de l'hospitalité et du refuge. C'est par une telle lecture rétrospective qu'il est possible de reconstituer une généalogie de retraites rousseauistes ${ }^{1}$, de reconstruire la fonction de son ultime isolement, l'ethos de cette décisive et finale expérience de la retraite. Grâce à la prise en compte de l'œuvre de Rousseau dans sa transversalité, l'absence d'écrits du philosophe en cette période n'empêche pas de reconstituer son expérience à Ermenonville, la consistance du bonheur qu'il éprouva à s'y retirer.

Mais pour atteindre la véracité de cette expérience, il est nécessaire de procéder à une lecture croisée de la Correspondance et de son œuvre autobiographique, chacune développant une philosophie spécifique de l'intime et relatant la chaîne des épisodes de sa vie. Par l'application d'une telle méthode généalogique, considérant Ermenonville comme la fin d'un parcours errant, comme le terme d'une fuite de refuges en asiles, il sera possible de comprendre ce qui se nomme aujourd'hui le parc Jean-Jacques Rousseau, et ses alentours, comme son dernier refuge, emblématique de son expérience de la retraite.

Qu'est-ce qui caractérise cette ultime retraite ? Peut-elle rétroactivement définir le renouveau du discours de la retraite qui se répand dans l'œuvre autobiographique de Rousseau ? Quel est le lien entre bonheur, retraite et expérience de l'intériorité ? La reconstitution du retirement à Ermenonville permet-il d'établir leur indissociabilité ?

\section{La retraite d'Ermenonville}

La dernière retraite de Jean-Jacques qualifie tout à la fois un lieu, Ermenonville, une période, somme toute assez courte - six semaines -, une attitude, celle de l'herborisation rêveuse, un état, solitaire, peut-être heureux, et enfin une dynamique, celle de l'isolement. Pour saisir les motifs, les enjeux et la teneur de cette dernière retraite, il est nécessaire de contextualiser son installation à Ermenonville, de saisir les relations entre Girardin et Rousseau, l'environnement que ce dernier projette pour ses derniers jours.

En février 1777, Rousseau écrit un mémoire pour trouver un refuge, afin d'aller couler à la campagne des jours heureux. Il souhaite goûter à nouveau aux charmes de la campagne et s'extraire définitivement des grandes villes. La situation, exposée de manière dramatique, présente l'offre d'un « asile » comme la seule solution à une situation devenue impossible à soutenir :

Réduits à vivre absolument seuls et néanmoins hors d'état de nous passer du service d'autrui, il ne nous reste dans les infirmités de l'abandon qu'un seul moyen de soutenir nos vieux jours. C'est de prier ceux qui disposent de nos destinées de bien vouloir disposer aussi de nos personnes et de nous ouvrir quelque asile où nous puissions subsister, à nos frais, mais exempts d'un travail qui désormais passe nos forces ${ }^{2}$.

\footnotetext{
${ }^{1}$ Sur les diverses retraites de Rousseau, voir l'art. d'A.-F. Grenon dans le présent numéro.

2 J.-J. Rousseau, Mémoire écrit au mois de février 1777, et depuis lors remis et montré à diverses personnes, dans Correspondance Complète, Janvier 1775-juillet 1778, t. XL, éd. R.-A. Leigh, Oxford, The Voltaire Foundation, 1982, p. 243.
} 
Selon Le Bègue de Presle, en février 1777, cela fait plus de huit mois déjà que Rousseau envisage une telle retraite ${ }^{3}$. À partir de la fin de cet hiver 1777 , on lui offre différents logements. Le Comte Duprat lui propose ainsi une maison aux environs de Clermont-Ferrand. Rousseau accepte puis renonce rapidement à cet «asile paisible et solitaire ${ }^{4}$ » en raison des incommodités du voyage qu'il craint pour aller de Paris jusqu'en Auvergne ${ }^{5}$. Le Prince de Ligne lui offre également un hébergement tentant ${ }^{6}$, puisqu'il conjugue à la présence de plusieurs beaux jardins disponibles pour les plantations, une maison commode et indépendante, ainsi qu'un environnement tel que Rousseau l'a toujours souhaité pour ses derniers jours : " Vous y aurez une petite maison de campagne, à vous tout seul, à un quart de lieue de la mienne; vous y planterez, vous y sèmerez, vous en ferez tout ce que vous voudrez [...] le ciel est beau, l'air y est pur $^{7}$.» Rousseau se décide finalement pour Ermenonville et s'y rend le 20 mai 1778. Une lettre du marquis de Girardin datée du 24 mai fait référence à cette installation : "Sachez que ces arcadians fields sont actuellement habités par le divin auteur d'Héloïse et d'Émile, il les a choisis pour sa retraite comme Apollon avait choisi La Vallée de Thessalie, et le véritable vaut mieux que le fabuleux ${ }^{8}$ ».

\section{«Mourir heureux » : caractéristiques de la retraite rousseauiste}

La trop grande proximité avec Paris rebute celui que l'inscription du Panthéon consacra comme «l'homme de la nature et de la vérité. » Ermenonville n'est pas trop proche d'une grande ville, et c'est certainement ce qui contribue à cette préférence. Même si la déclaration suivante n'est pas effectuée à propos d'Ermenonville, elle éclaire l'une des dernières décisions de Jean-Jacques, qui a toujours eu les mêmes critères tout au long de sa vie pour choisir un lieu retiré où il pourra être en sûreté : «En attendant j'ai été faire une promenade dans la Province de Surrey où j'ai été extrêmement tenté de me fixer ; mais le trop grand voisinage de Londres, ma passion croissante pour la retraite et je ne sais quelle fatalité qui me détermine indépendamment de la raison m'entraînent dans les montagnes du Derbishire ${ }^{9}$. » Les critères sont purement affectifs ; la retraite est l'objet d'un engouement et assure au promeneur solitaire d'assouvir sa passion pour la botanique.

L'environnement est toujours au moins aussi important que le logement en lui-même ; même si à l'époque où il cherche une dernière retraite, il souhaite en priorité un séjour tranquille, pouvant contribuer à son bonheur ${ }^{10}$. Il faut à Rousseau un climat tempéré et une nature susceptible de satisfaire son penchant pour les courses botaniques solitaires. Aussi refuse-t-il déjà en 1766 un logement situé sur l'île de Wight en raison de la pauvreté de l'environnement naturel.

\footnotetext{
3 «Il y a plus d'un an que M. Rousseau disait qu'il voulait se retirer à la campagne » (Louis Le Bègue de Presle, ibid., p. 327).

${ }^{4}$ J.-J. Rousseau, Lettre à M. le Comte Duprat du 31/12/1777, n ${ }^{\circ}$ 7148, ibid., p. 184.

5 «Je désire d'aller finir mes jours dans l'asile aimable que vous voulez bien me destiner, [...] le mal est qu'il faut s'y transporter » (Lettre à M. le Comte Duprat du 3/02/1778, n 7155, ibid., p. 194).

${ }^{6}$ Rousseau n'accepte pas cette proposition parce qu'il «décèle dans l'offre de Ligne une manœuvre de ses ennemis » (Raymond Trousson, «Un visiteur de la rue Plâtrière : le Prince Charles-Josephde Ligne », dans Rousseau visité, Rousseau visiteur : les dernières années (1770-1778), Actes du colloque de Genève (21-22 juin 1996), éd. Michel Porret et Jacques Berchtold, Annales de la société Jean-Jacques Rousseau, t. XVII, Genève, Droz, 1999, p. 241). Rousseau n'a pas répondu par une lettre à l'offre de Ligne mais ce dernier confirmera la méfiance de Jean-Jacques à son égard : «Il eut la bonté de croire, à sa façon ordinaire, que les offres d'asile que je lui faisais étaient un piège que ses ennemis m'avaient engagé à lui tendre » (Prince de Ligne, Lettres et pensées du maréchal prince de Ligne, cité dans R. Trousson, ibid., p. 242).

${ }^{7}$ Charles-Joseph Prince de Ligne, Lettre à J.-J. Rousseau du 01/05/1778, n 4143, Correspondance Générale, éd. Théophile Dufour, Armand Colin, 1934, t. XX, p. 340.

${ }^{8}$ René-Louis de Girardin, Lettre à un cousin italien, ${ }^{\circ}$ 7168, t. XL, C. C., p. 218.

${ }^{9}$ Lettre à M. Du Peyrou du 14/03/1766, nº 5103, t. XIX, C. C., p. 28.

10 «L'aménité du sol et les agréments du paysage ne sont plus pour moi des objets à mettre en balance avec un séjour tranquille et la bienveillante hospitalité » (Lettre à M. le Comte Duprat du 31/12/1777, nº 7148, t. XL, C. C., p. 184).
} 


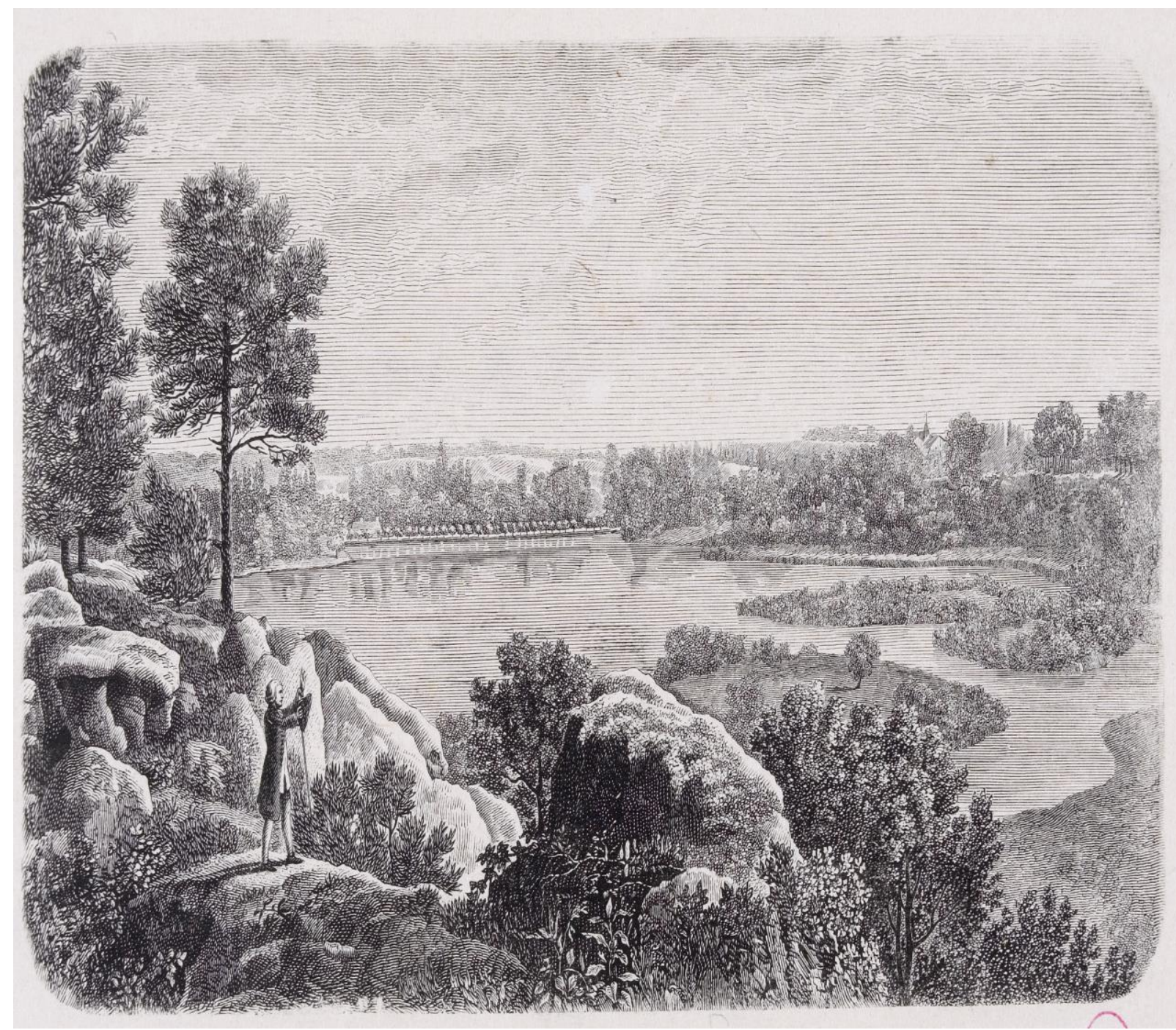

Julien-Antoine Peulot, Jean-Jacques Rousseau à Ermenonville, estampe, musée du domaine départemental de Sceaux, photographie de Benoît Clain, XIX ${ }^{\mathrm{e}}$ siècle.

Il est également nécessaire qu'un lieu de refuge soit propice à la solitude. C'est au terme d'une errance interminable qu'il fait d'Ermenonville sa dernière retraite, après avoir connu en sa vie de très nombreux asiles : "J'ai beau soupirer après la solitude dont j'ai besoin dans mon état; je ne puis la trouver nulle $\operatorname{part}^{11}$. » À propos de Wootton, refuge solitaire d'Angleterre, il écrivait à l'époque à Du Peyrou : "Après tant de fatigues et de courses $\mathrm{j}$ 'arrive enfin dans un asile agréable et solitaire où $\mathrm{j}$ 'espère pouvoir respirer en paix ${ }^{12}$. » La formule surgit fréquemment dans sa Correspondance: "Cet asile [...] que j'habite est agréable et solitaire ; $j$ 'espère $y$ pouvoir couler paisiblement le reste de mes malheureux jours ${ }^{13} »$.

En Angleterre, il reçoit de nombreuses propositions d'hospitalité et opte pour celle qui pourrait emporter sa préférence. Le parallèle est d'autant plus flagrant entre l'asile de Wootton et celui d'Ermenonville qu'il comporte plus d'une marque de similitude. La déclaration suivante aurait pu être celle de Rousseau à Ermenonville, ce qui montre à quel point sa philosophie de la retraite reste constante :

\footnotetext{
${ }^{11}$ Lettre à M. Rey du 3/03/1766, nº 5089, t. XXIX, C. C., p. 10.

${ }^{12}$ Lettre à M. Du Peyrou du 29/03/1766, n ${ }^{\circ}$ 5134, ibid., p. 71.

${ }^{13}$ Lettre à Mme Boy de la Tour du 9/04/1766, n 5153, ibid., p. 105.
} 
L'affluence me fait trouver le séjour de la ville incommode. Aussitôt les maisons de campagne se présentent en foule; on m'en offre à choisir dans toutes les provinces [...] j'hésite longtemps sur le choix, je me détermine enfin [...] je pars ; j'arrive dans une habitation commode, agréable et solitaire : le maître prévoit tout, rien ne manque ; je suis tranquille, indépendant ${ }^{14}$.

Un asile où il se sent à la fois heureux et protégé de toute malveillance est en même temps un lieu où il envisage de mourir ${ }^{15}$ :

C'est beaucoup sans doute de trouver sur la terre un endroit où l'on me laisse : mais si j'en trouve en même temps un où je me plaise n'est-ce pas encore plus? Si je vais dans l'île de Wight j'en voudrai sortir ; mais si je vais au pays de Galles j’y voudrai mourir ${ }^{16}$.

S'il est si soigneux dans le choix de ces habitations, c'est parce qu'il souhaite « [...] vivre et mourir en repos ${ }^{17}$. » À la fin de son existence, il formulera à nouveau ce souhait de mourir heureux, dans un environnement propice. Il faut pour ce faire satisfaire une dernière dynamique de l'isolement, être seul et loin de la foule des hommes, ce qui sera le cas à Ermenonville : «Quand je ne verrai plus [les hommes] j'oublierai bientôt leur haine, et cet oubli m'est nécessaire pour vivre et mourir en paix ${ }^{18} »$.

\section{Expériences de l'hospitalité}

Rousseau a le projet dès 1768 de finir ses jours non en Angleterre ni en Suisse, mais en France. L'invitation du Marquis de Girardin est donc comme l'écho inversé d'une nécessité vitale ressentie par l'homme errant dix ans auparavant : «Celui de France est de tous les airs du monde celui qui convient le mieux à mon corps et à mon cœur; et, tant qu'on me permettra d'y vivre en liberté, je ne choisirai point d'autre asile pour finir mes jours ${ }^{19}$. » Lorsque l'auteur des Confessions fait référence à une demande d'hospitalité, c'est toujours en tenant pour acquis que la quête d'hospitalité est une recherche du bonheur. Les critères à partir desquels il fait ses choix de refuge ne diffèrent pas selon les époques. Douze ans plus tôt, lorsqu'il est en Angleterre, il cherche une retraite pouvant lui assurer le bonheur du repos, de la paix et de la tranquillité : «Je soupire incessamment après cet asile paisible [du pays de Galles] où l'on me promet le repos et dont si je l'y trouve, je ne sortirai jamais ${ }^{20}$. » Un refuge propice est pour lui «lieu de $\operatorname{repos}^{21}$ » pouvant générer le bonheur de la tranquillité : «Je compte [...] goûter dans la retraite que j'aime le calme et la paix dont j'ai si grand besoin et à quoi je borne désormais le bonheur de ma vie ${ }^{22} »$.

Parmi les marques d'amitié, c'est à l'hospitalité qu'il est le plus sensible : chassé de France, expulsé de l'île de Saint-Pierre, malvenu sur le territoire suisse et dans sa ville natale, il cherche en 1765 asile et protection. C'est ce que Hume lui propose, sans le connaître autrement que par ses écrits et les relations qu'ils ont en commun. Le solitaire errant est alors particulièrement touché par ce témoignage d'amitié, lui qui aurait presque souhaité, par dépit, être incarcéré plutôt que persécuté et pourchassé ${ }^{23}$. Prodiguée de manière cordiale ou

\footnotetext{
${ }^{14}$ Lettre à M. de Malesherbes du 10/05/1766, n 5195, ibid., p. 189.

15 «Vous savez ce qu'il me faudrait de plus pour mourir heureux [...]. En attendant j’herborise je me promène ». (Lettre à $M$. Du Peyrou du 21/06/1766, $\mathrm{n}^{\circ}$ 5236, ibid., p. 266).

${ }^{16}$ Lettre à Mme la Comtesse de Boufflers du 6/02/1766, $\mathrm{n}^{\circ}$ 5036, t. XXVIII, C.C., p. 275.

${ }^{17}$ Lettre à M. Guy du 2/08/1766, n 5332, t. XXX, C.C., p. 197.

${ }^{18}$ Lettre à M. le Comte Duprat du 31/12/1777, n 7148 , t. LX, C. C., p. 184.

${ }^{19}$ Lettre à M. le Marquis de Mirabeau du 28/01/1768, n ${ }^{\circ}$ 6222, t. XXXV, C. C., p. 58.

${ }^{20}$ Lettre à Mme la Comtesse de Boufflers du 6/02/1766, n ${ }^{\circ}$ 5036, t. XXVIII, C. C., p. 275.

${ }^{21}$ Lettre à Mme la Comtesse de Boufflers du 5/04/1766, nº 5146, t. XXIX, C. C., p. 92.

${ }^{22}$ Lettre à M. Roguin du 15/02/1766, n 5056, t. XXVIII, C. C., p. 304.

23 «J'osai désirer et proposer qu'on voulût plutôt disposer de moi dans une captivité perpétuelle que de me faire errer incessamment sur la terre en m'expulsant successivement de tous les asiles que j'aurais choisis » (J.-J. Rousseau, Les Confessions, XII, Euvres complètes, Paris, Gallimard, 1959, t. I, p. 647. Dorénavant : Confessions, XII, p. 416).
} 
bienveillante, l'hospitalité est motivée par l'amitié, et Rousseau n'envisage pas l'une sans l'autre : «[...] il vous offre sa maison. L'innocence et la paix y règnent ; vous y trouverez l'amitié, l'hospitalité, l'estime, la confiance. Consultez votre cœur, et s'il n'y a rien là qui vous effraie, venez sans crainte. Vous ne partirez point d'ici sans y laisser un $\operatorname{ami}^{24} \gg$.

Souvent, l'hospitalité représente le terme de l'errance pour le promeneur, comme il le narre dans les premiers livres des Confessions. Errer est parfois une manière volontaire de se perdre, afin de trouver une maison vraiment hospitalière et d'être l'objet de la bonté de gens simples et non policés ${ }^{25}$. Rousseau réitérera ces expériences heureuses de l'errance comme quête de l'hospitalité désintéressée, et ce jusqu'à sa mort. Mais il fuira tout hébergement intéressé, fondé sur la politesse, l'urbanité ou les mondanités, exigeant implicitement un contre-don, un « contrat ${ }^{26}$ » tacite funeste au bonheur de la liberté et de l'indépendance. Toute expérience heureuse de l'hospitalité ${ }^{27}$ implique au contraire de ne pas se sentir redevable, d'être par conséquent reçu gracieusement, par charité, "vrai bienfait ${ }^{28}$ », libéralité ou amitié, en toute simplicité, avec autant de bienveillance, d'intimité et de discrétion que possible.

Des premières expériences de l'hospitalité narrées dans Les Confessions aux dernières herborisations du promeneur solitaire à Ermenonville, la réflexion sur l'asile et le refuge reste constante, et la retraite est invariablement conçue comme une condition du bonheur.

\section{Retraite volontaire, exil subi}

Une retraite garantit bien évidemment la sécurité de celui dont la maison fut lapidée à Môtiers ${ }^{29}$ et n'est toujours quittée qu'à regret : «[...] notre départ m'avaient donné un grand intérêt pour la sûreté de notre retraite [...] je ne faisais pas un pas sans sentir que je m'éloignais de ce bonheur ${ }^{30}$. » Le départ d'un refuge n'est souvent que forcé et contraint. Lorsqu'il avait trouvé asile chez Mme de Warens, Rousseau avait également doublé cette retraite d'une autre encore plus reculée, en l'occurrence un petit jardin, "retraite champêtre $^{31} »$, au cas où des visiteurs viendraient troubler la sérénité qu'il cultive avant tout ${ }^{32}$. Il est donc patent que la retraite est choisie positivement par Rousseau. S'isoler des autres est un choix assumé, une préférence et une inclination du cœur pour la vie solitaire, à la campagne. À ce titre, la dynamique de l'isolement et la situation de solitude qui en résulte sont librement choisis. En revanche, le changement incessant de lieu de retraite lui est imposé. À partir de $1756^{33}$, sa vie prend une tournure de plus en plus errante, il devient un écrivain sans domicile fixe, un expulsé cherchant refuge :

En quelque asile que je voulusse me réfugier [...] je ne pouvais donc compter sur aucune retraite assurée $[\ldots]$ me faire errer incessamment sur la terre, en m'expulsant successivement de tous les asiles que j'aurais choisis ${ }^{34}$.

\footnotetext{
${ }^{24}$ J.-J. Rousseau, La Nouvelle Héloïse, Euvres complètes, éd. cit., t. II, IV, 4, 1964, p. 416.

25 «Un jour entre autres m'étant à dessein détourné [...] j'entrai chez un paysan [...] en état d'exercer l'hospitalité [...] et je fis un dîner tel qu'autre qu'un piéton n'en connut jamais » (Confessions, IV, p. 163-164).

26 « Je sais qu'il y a une espèce de contrat et même le plus saint de tous entre le bienfaiteur et l'obligé » (Les Rêveries du promeneur solitaire, Euvres complètes, éd. cit., t. I, II, p. 1053. Dorénavant : Rêveries, II, p. 1053).

${ }^{27}$ Guilhem Farrugia, Bonheur et fiction chez Rousseau, Paris, Classiques Garnier, «L'Europe des Lumières », n 19, 2012, p. 265-280.

28 «Je ne reconnais pour vrais bienfaits que ceux qui peuvent contribuer à mon bonheur et c'est pour ceux-là que je suis pénétré de reconnaissance; mais certainement l'argent et les dons n’y contribuent pas » (J.-J. Rousseau, Mon Portrait, Euvres complètes, t. I, 1959, éd. cit., p. 1126).

${ }^{29}$ « Tandis que je balançais ainsi, vinrent les persécutions de Môtiers, qui me forcèrent à la retraite » (ibid., XII, p. 651-652).

${ }^{30}$ Ibid., III, p. 130

${ }^{31}$ Ibid., V, p. 223.

32 «Mais elle était si souvent entourée, et de gens qui me convenaient si peu, que le dépit et l'ennui me chassaient dans mon asile » (ibid., V, p. 182).

${ }^{33}$ «Ce fut le 9 avril 1756 que je quittai la ville pour ne plus y habiter» (ibid., IX, p. 403).

${ }^{34}$ Ibid., XII, p. 647.
} 
En 1776, il ne conçoit plus la fin de sa vie que comme une «longue retraite dans laquelle [il] doi $[\mathrm{t}]$ achever $[\mathrm{s}] \mathrm{es}$ jours ${ }^{35}$. » Il mourra à Ermenonville, sa dernière retraite : «Jean-Jacques passa les derniers mois de sa vie à Ermenonville, où le Marquis de Girardin, son fervent admirateur, lui avait offert refuge, dans sa propriété aménagée à l'exemple du jardin de La Nouvelle Hélö̈se $e^{36} »$.

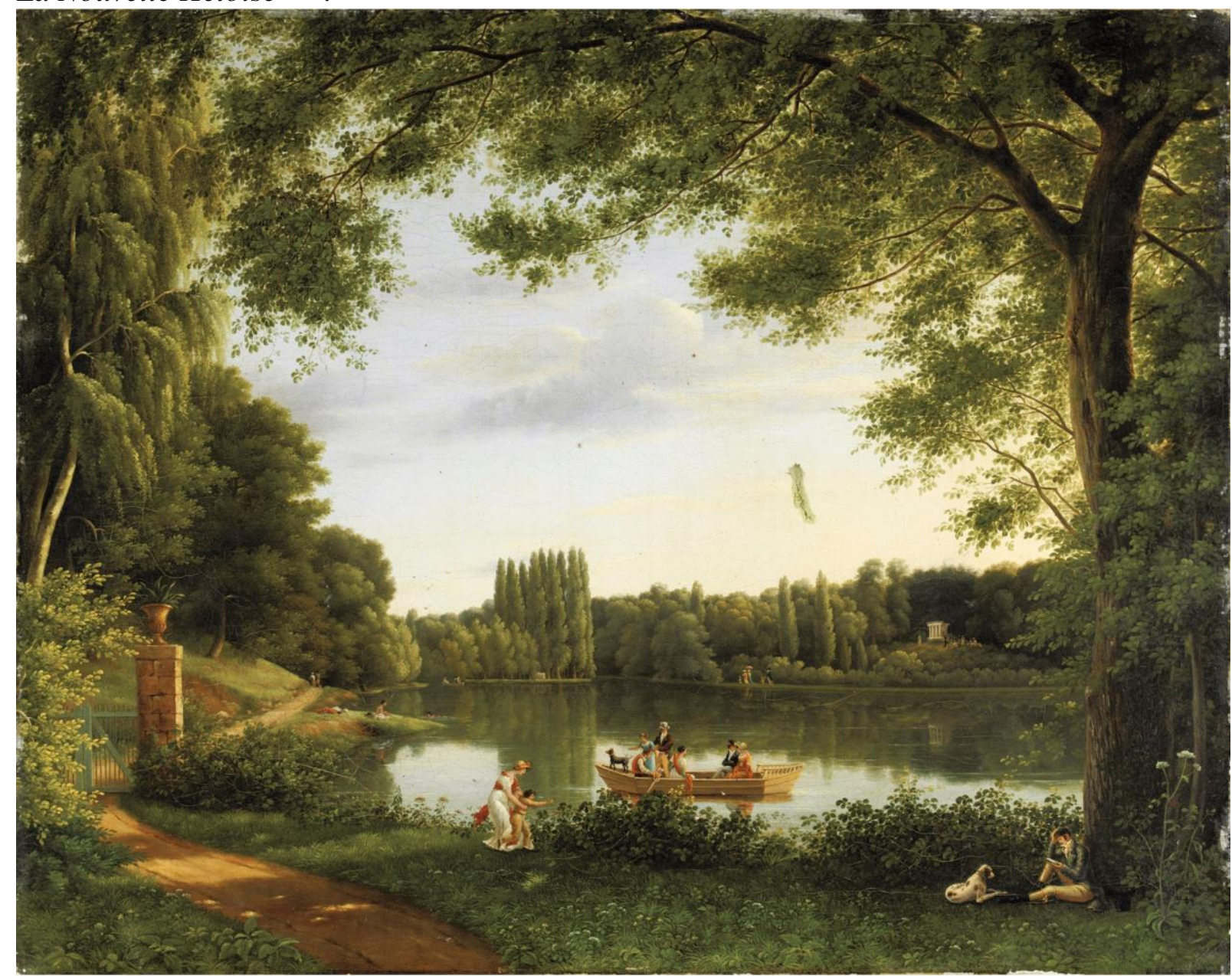

Jean-Joseph-Xavier Bidauld, Vue du lac d'Ermenonville, huile sur canevas, collection particulière, photographie de Sotheby's, XVIII' siècle.

Si l'action de se retirer du monde n'est pas expérimentée comme un exil, c'est parce qu'il se décentre de Paris, de la «coterie holbachique», de la cour et des cercles littéraires et mondains. Avant d'être perverti par la société, avant que l'habitude des mondanités dégénère en un «vrai besoin ${ }^{37}$ », il prend soin de se recentrer, de «chercher le calme et la paix dans la retraite» afin de trouver dans sa propre sphère intérieure les ressources de son bonheur. Rousseau assigne à l'âme du solitaire une forme circulaire et concentrique. Il s'agit pour être heureux de se circonscrire en soi-même ${ }^{38}$, à l'image de l'île de Bienne au centre du lac, à l'image également de «l'insecte au centre de sa toile ${ }^{39}$. » L'amour de ce lac provient d'une

\footnotetext{
${ }^{35}$ Rêveries, I, p. 1004.

${ }^{36}$ Bronislaw Baczko, Rousseau, solitude et communauté, Paris, École Pratique des Hautes Études, 1974, p. 397.

37 «C'est que la longue habitude de vivre à la cour devenait un vrai besoin [...] et que la retraite que je lui conseillais serait moins un repos pour lui qu'un exil » (Confessions, XI, p. 552).

38 "Commençons par redevenir nous, par nous concentrer en nous [...] commençons en un mot par nous rassembler » (J.-J. Rousseau, Lettres morales, Euvres complètes, t. IV, 1964, éd. cit., p. 1112).

39 « Mesurons le rayon de notre sphère et restons au centre comme l'insecte au centre de sa toile, nous nous suffirons toujours à nous-mêmes » (J.-J. Rousseau, Émile, ou de l'éducation, II, éd. cit., p. 305).
} 
conception du bonheur comme circonscription en soi-même et comme circulation dans sa propre circularité intérieure. Cet espace resserré et délimité est le duplicata de l'intériorité du solitaire. Par là même, il répond parfaitement aux exigences formulées dans l'Émile: «Ô homme ! Resserre ton existence au-dedans de toi, et tu ne seras plus misérable ${ }^{40}$. » En découvrant le lac de Bienne, Rousseau rencontre donc son alter ego naturel, et souhaite vivre dans cette île comme il désire vivre en lui-même. L'île et le moi du solitaire forment deux cosmos qui sont régis par les mêmes lois, semblables en ceci qu'ils sont séparés du reste du monde, indépendants et autonomes par rapport à celui-ci ${ }^{41}$. Lorsqu'il conduira sa barque « au milieu du lac », il trouvera en cet endroit abrité, délimité et sûr, perçu comme un asile, les conditions propices à une «plongée » dans l'intériorité, à force de cultiver "l'habitude de rentrer en soi ».

\section{Retraite et bonheur}

Le discours sur la retraite est un discours du bonheur. Sans félicité, pas de consécration moderne de l'intériorité, pas de renouveau de l'idée de retraite. Cette notion de retraite n'acquiert une valeur existentielle qu'à partir du moment où l'idée de salut est remplacée par celle de bonheur ${ }^{42}$. C'est bien l'expérimentation d'un bonheur ici-bas, bien distinct d'un salut dans la vie à venir qui génère une nouvelle philosophie de la retraite. Plus qu'un simple lieu, la retraite devient alors une attitude ${ }^{43}$, un état ${ }^{44}$, un mouvement ${ }^{45}$, une temporalité ${ }^{46}$.

C'est la retraite qui garantit l'attention à l'intériorité, qui est le terreau favorable à l'introspection, au terme de laquelle le bonheur peut être atteint. La cinquième promenade des Rêveries du promeneur solitaire, consacre ce bonheur intérieur, accessible dans la retraite extatique du lac de Bienne. Il s'agit, en recherchant une retraite, de trouver, non pas un point de fixation extérieur mais une «assiette ${ }^{47}$ » intérieure constitutive de la félicité. La dynamique de l'isolement n'est pas seulement matérielle et géographique ; elle est également intérieure et repose sur la découverte d'un espace interne, sur une retraite interne, lorsque le « cœur [...] se retire au-dedans de lui ${ }^{48}$. » Le déplacement matériel et extérieur engendre une mobilité spirituelle et intérieure. Le bonheur de marcher relève donc de l'aménagement d'un espace, à saisir dans sa dualité : espace extérieur, environnement naturel, lieu de déambulations, de résidences, de promenades, de repos, de refuges, d'asiles, dont Ermenonville est le dernier, mais aussi espace intérieur, espace intime de la méditation et de la rêverie.

C'est la découverte moderne de l'intériorité qui donne par conséquent un espace au bonheur de l'individu, qui lui permet de déployer sa félicité en se fixant sur une étendue spatiale, toute intérieure et personnelle. Le « vrai bonheur » est en effet un état «parfait, suffisant et plein », caractérisé par la plénitude d'une âme qui, «remplie tout entière » du seul sentiment de l'existence, cherche la stabilité d'un espace où elle puisse se «reposer tout entière et

\footnotetext{
${ }^{40}$ Ibid., p. 308.

${ }^{41}$ «Dans une île fertile et solitaire, naturellement circonscrite et séparée [...] (Rêveries, V, p. 1048).

42 «Les Lumières, en particulier françaises, substituent aux valeurs transcendantes des valeurs immanentes (le bonheur s'oppose au salut) » (Michel Delon, art. BonHeur, dans Dictionnaire européen des Lumières, dir. M. Delon, Paris, PUF, Quadrige, Dicos poche, 2007, p. 190.

${ }^{43}$ Voir à ce sujet l'ouvrage de Pierre Burgelin, La Philosophie de l'existence de J.-J. Rousseau, dans lequel celui-ci qualifie l'attitude liée à l'expérience de la retraite : «Pour chercher le bonheur [1'âme] doit premièrement se trouver, donc se replier sur soi, y adhérer et en jouir » (Genève, Slatkine reprints, 1978 (1 $1^{\mathrm{e}}$ éd. 1952), p. 149).

${ }^{44} \mathrm{Si}$ la solitude est un état heureux excluant toute agitation, cet état repose néanmoins sur une dynamique, celle de se retirer de la vie sociale. Avant d'être seul, il faut d'abord s'inclure dans un processus de retraite.

${ }^{45} \mathrm{Il}$ s'agit alors de l'action de se retirer, de la dynamique de l'isolement qui permet d'expérimenter le fait d'être retiré, la situation de solitude actualisée : «Mon premier mouvement fut de me retirer de Genève [...] 》 (Confessions, XI, p. 581).

${ }^{46}$ Plutôt qu'une propriété objective des choses qui affecte l'âme, le temps devient intérieur et éprouvé, une propriété de l'âme elle-même. L'existence devient un «sentiment », le temps une donnée intérieure et subjective et la retraite une période plus ou moins longue consacrée au repos, à la solitude, permettant de jouir du sentiment actuel de l'existence.

${ }^{47}$ Rêveries, V, p. 1046.

${ }^{48}$ Lettre à M. De Luc du 24/02/1765, n 4059, t. XXIV, C. C., p. 87.
} 
rassembler là tout son être ${ }^{49}$. » L'exigence rousseauiste de se concentrer, de se regrouper et de se circonscrire en soi-même présuppose la découverte d'un espace intérieur sphérique et équilibré nécessaire au bonheur ${ }^{50}$.

Le bonheur suppose une situation solitaire, propice à la paix de l'âme, à la constance et à la durée du sentiment heureux, ainsi qu'à l'autonomie d'une vie indépendante ${ }^{51}$. C'est pour favoriser ce sentiment que Rousseau et Mme de Warens aménagent déjà aux Charmettes, « terre [...] retirée et solitaire », propice à la tranquillité de l'âme. C'est en se fixant dans cet « asile un peu sauvage et retiré » qu'il trouvera la félicité ${ }^{52}$.

Il réitérera l'expérience à l'Ermitage, que Mme d'Épinay lui fait aménager et lui offre : « Mon ours, voilà votre asile ${ }^{53}$.» Tout refuge est en ce sens pour Rousseau un lieu de bonheur : «Ah ! Madame, quelle habitation délicieuse ! Voilà un asile tout fait pour moi ${ }^{54}$. » À noter qu'un refuge heureux ne peut être urbain. Il est au contraire systématiquement trouvé à la campagne ${ }^{55}$, et c'est seulement dans un environnement naturel que le bonheur peut être éprouvé :

$[\ldots]$ je soupire après la campagne et la solitude $[\ldots]$ avant que $\mathrm{j}$ 'aie atteint l'asile que je vois chercher $[\ldots]$ sitôt que je me vois sous les arbres, au milieu de la verdure, je crois me voir dans le paradis terrestre et je goûte un plaisir interne aussi vif que si j'étais le plus heureux des mortels ${ }^{56}$.

Du bonheur de l'amour ${ }^{57}$ à celui de la plus douce amitié s8 $^{58}$, le bonheur en société restreinte prend avant tout racine dans une retraite, permettant une vie tranquille et privée, séparée du tumulte du monde. Une retraite est également un lieu propice au bonheur de méditer ${ }^{59}$. Un « lieu solitaire ${ }^{60}$ » donne de l'élan à la pensée : «Je m'engouais de cette petite retraite [...] pour y penser avec plus de plaisir ${ }^{61}$. » En se retirant du monde, Rousseau se livre tantôt, non à l'oisiveté ${ }^{62}$ mais tout au contraire une situation propice au « travail ${ }^{63}$ », tantôt aux délices de la paresse, aux «douceurs du désœuvrement et de la vie contemplative ${ }^{64}$. »C'est le fait d'être retiré qui génère tout autant le bonheur de l'imagination : «Plus j'examinais cette charmante retraite, plus je la sentais faite pour moi. Ce lieu solitaire plutôt que sauvage me transportait en idée au bout du monde ${ }^{65}$. $\gg$ La félicité apparaît en ce sens comme la visée de la retraite ${ }^{66}$.

\footnotetext{
${ }^{49}$ Rêveries, V, p. 1046.

50 «Mon cœur, uniquement occupé du présent, en remplit toute sa capacité, tout son espace » (Confessions, III, p. 130-131).

51 «Me voilà donc enfin chez moi, dans un asile agréable et solitaire, maitre d'y couler mes jours dans cette vie indépendante, égale et paisible, pour laquelle je me sentais né » (ibid., IX, p. 413).

$52 \ll[\ldots]$ ce séjour est celui du bonheur et de l'innocence [...]» (ibid., p. 224).

${ }^{53}$ Ibid., VIII, p. 396.

${ }^{54} I d$.

55 «Je me sentais fait pour la retraite et la campagne ; il m'était impossible de vivre heureux ailleurs » (ibid., IX, p. 401).

${ }^{56}$ Rêveries, VIII, p. 1083.

57 «[...] nous établir dans une solitude agréable [...] [qui] nous eût vraisemblablement assuré des jours heureux et tranquilles jusqu'au moment où la mort devait nous séparer »(Confessions, V, p. 223).

58 «M. Mussard [...] s'était fait à Passy une retraite charmante où j'ai coulé de bien paisibles moments », (ibid., VIII, p. 373).

59 «L'ouvrage que j'entreprenais ne pouvait s'exécuter que dans une retraite absolue; il demandait de longues et paisibles méditations que le tumulte de la société ne souffre pas » (Rêveries, III, p. 1015).

${ }^{60}$ Confessions, IX, p. 403.

${ }^{61}$ Ibid., V, p. 181.

$62 \ll[\ldots]$ je puis dire que ce temps, où je vivais dans la retraite et toujours malade, fut celui de ma vie où je fus le moins oisif et le moins ennuyé » (ibid., VI, p. 235).

${ }^{63}$ «Cette vie retirée devint avantageuse à mon travail» (ibid., VII, p. 333).

${ }^{64}$ «Tel était l'asile que je m'étais ménagé [...]. Ce choix était conforme à mon goût pacifique, à mon humeur solitaire et paresseuse [...] aux douceurs du désœuvrement et de la vie contemplative » (ibid., XII, p. 638).

${ }^{65} \mathrm{Ibid}$., IX, p. 403.

${ }^{66}$ « Je n'avais plus de goût à rien qu'à [...] faire sentir en quoi consistait le vrai charme d'une vie heureuse [...] à la campagne » (ibid., V, p. 223).
} 


\section{Mythologie d'un sanctuaire du bonheur}

Selon certains témoins de la fin de la vie de Jean-Jacques, celui-ci aurait coulé à Ermenonville des jours heureux. Le Bègue de Presle tend à nous donner des indications en ce sens lorsqu'il écrit, le 3 juillet 1778 : «La fatale destinée de Monsieur Rousseau vient de l'enlever à la paix et au bonheur qu'il avait trouvé dans la retraite auprès de personnes aussi tendres que lui ${ }^{67}$. » Celui-ci rédige même dès juillet 1778 un petit mémoire intitulé Un dîner à Ermenonville, où il fait le portrait de Jean-Jacques lors de ses dernières semaines, et le décrit comme un homme heureux, herborisant comme à l'ordinaire, content de son sort : «J'entrai en conversation avec M. Rousseau ; et je fus on ne peut plus enchanté de le voir dans un état paisible, et tout à fait à son aise. La tranquillité de son âme et le contentement de son cœur se produisaient sur son visage et dans ses discours ${ }^{68} \gg$.

L'aménagement du parc d'Ermenonville répond à un projet paysagiste pittoresque visant à produire le bonheur sensitif du promeneur ${ }^{69}$; il s'inscrit également dans un projet de petite société heureuse. Dès l'introduction de son traité précité, Girardin affirme qu' « un jardin [...] fut le premier séjour de l'homme heureux » et que son "effet doit répandre la sérénité dans l'âme ${ }^{70} »$, de manière à «[...] jouir avantageusement des paysages ${ }^{71}$. » Le parc d'Ermenonville doit produire a minima le «contentement ${ }^{72} »$ de celui qui assiste à son spectacle, et si possible le «bonheur ${ }^{73} »:$ : «...] ces fils délicats, à l'extrémité desquels tiennent le bonheur et le bien-être [...]. Rien n'est plus intéressant que de prévenir la souffrance, et de procurer le bonheur ${ }^{74}$. »Ce qu'il nomme «le jardin paysage » est aménagé pour produire le bonheur de l'individu, pour générer « [...] le goût des véritables jouissances de la nature, des plaisirs purs exempts de regrets, et le spectacle de campagnes heureuses ${ }^{75}$. » Rousseau passa vraisemblablement ${ }^{76}$ ses derniers jours à se promener et herboriser, en particulier dans des lieux déserts, comme il le déclare déjà en 1777 dans les Rêveries : «Le plaisir d'aller dans un désert chercher de nouvelles plantes couvre celui d'échapper à mes persécuteurs ${ }^{77}$. $\gg$ Ce désert, il le trouvera à Ermenonville. La zone située à l'ouest du domaine et faisant partie du parc initial, où Rousseau se plaisait à herboriser, a d'ailleurs été nommée « le Désert » par le Marquis.

Après la mort de Rousseau, Girardin fait ériger un tombeau ${ }^{78}$ dans l'île des peupliers et célèbre plus que jamais la mort du philosophe.

Il contribue à ce culte qui s'installe et qui fait d'Ermenonville un lieu de retraite et de pèlerinage. L'iconographie la plus reproduite sur Rousseau est probablement aujourd'hui celle qui le représente herborisant à Ermenonville.

\footnotetext{
${ }^{67}$ Louis Le Bègue de Presle, Lettre à J.-J. Rousseau du 3/07/1778, nº 7180, t. LX, C. C., p. 236.

${ }^{68}$ Louis Le Bègue de Presle, «Un dîner à Ermenonville », ibid., p. 323.

${ }^{69}$ Sur la dimension sensorielle de la retraite, voir Hélène Cussac, «Espace et Bruit. Le monde sonore dans la littérature française du $18^{\mathrm{e}}$ siècle », L'information littéraire, Paris, Belles-Lettres, $\mathrm{n}^{\circ}$ 2, 2006, p. 46-50.

${ }^{70}$ Girardin, De la composition des paysages, ou des moyens d'embellir la nature, Genève, Delaguette, 1977 [1777], p. 2.

${ }^{71}$ Ibid., p. 78.

72 Ibid., p. 137.

73 « La cabane $[\ldots]$ cette retraite $[\ldots]$ est habitée par le bonheur » (ibid., p. 133).

${ }^{74}$ Ibid., p. 151.

${ }^{75}$ Ibid., p. 158

76 «Quelles qu'aient pu être les affabulations des Girardin, père et fils, sur un séjour qui allait durer le même temps que celui de l'île de Saint-Pierre, et sur sa mort, on ne peut douter que Rousseau y ait herborisé » (Henry Chéron, art. BOTANIQUE, Dictionnaire de Jean-Jacques Rousseau, dir. Raymond Trousson et Frédéric Eigeldinger, Paris, Champion, 2001, p. 105).

${ }^{77}$ Rêveries, VII, p. 1070.

78 «Mort de Rousseau le 2 juillet 1778. Il est enterré le 4 juillet dans l'île des peupliers, une île artificielle élevée au milieu du parc [...]. Un premier monument surmonté d'une urne fut d'abord érigé : il figure sur une gravure célèbre de Moreau le Jeune [...]. En 1780, un autre monument de style antique, dessiné par Hubert Robert et sculpté par J.-P. Lesueur, le remplaça » (Tanguy L'Aminot, art. ERmenOnVILle, Dictionnaire de Jean-Jacques Rousseau, ouvr. cité, p. 305-308).
} 


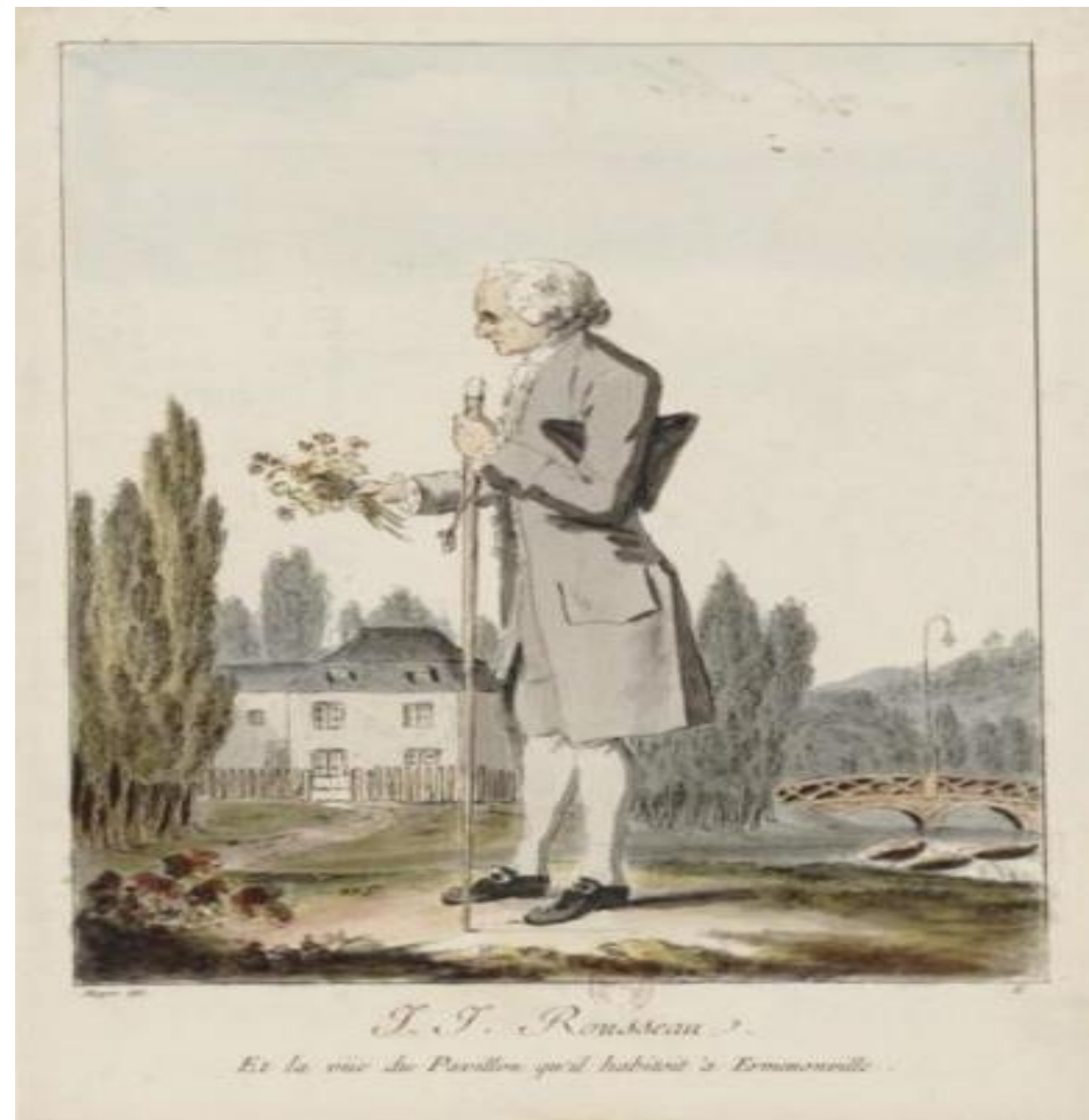

Georges-Frédéric Meyer, Jean-Jacques Rousseau herborisant à Ermenonville, aquarelle, musée Carnavalet, photographie de la Bibliothèque Nationale de France, XVIII ${ }^{\mathrm{e}}$ siècle.

Son séjour n'y fut pourtant que de six semaines. Mais sa dernière retraite acquiert après sa mort une dimension emblématique et symbolique. Avant son intronisation au Panthéon ${ }^{79}$, le parc devient le lieu où l'on vient rendre hommage au célèbre penseur du siècle des Lumières, dont «l'histoire va se muer en essence ${ }^{80}$. » Une partie du domaine deviendra au $20^{\mathrm{e}}$ siècle le «Parc Jean-Jacques Rousseau ${ }^{81} »$ en raison de la portée symbolique de ce pèlerinage. Le «mythe ${ }^{82} »$ est en construction, Ermenonville symbolise alors la retraite comme savoir-vivre ; il devient l'emblème de l'asile heureux.

Guilhem FARRUGIA Université de Poitiers, FoRell

\footnotetext{
${ }^{79}$ Rousseau entra au Panthéon le 10 octobre 1794. Le cercueil avait été remis au Marquis de Girardin le 8 octobre 1794.

${ }^{80}$ Robert Mauzi, L'Idée du bonheur dans la littérature et la pensée françaises au XVIII siècle, Paris, Slatkine, 1979 ( $1^{\mathrm{e}}$ éd. 1960), p. 508.

${ }^{81}$ Cette dénomination serait effective depuis 1938, date de l'acquisition du parc par le Touring-Club de France.

${ }^{82}$ Bronislaw Baczko, ouvr. cité, p. 397.
} 
Université Paris 4 Sorbonne, CELLF $17^{e}-18^{e}$ siècles 